\title{
Analyse de la distribution spatiale des Acanthaceae en Afrique Centrale et comparaison avec les théories phytogéographiques de Robyns, White et Ndjele.
}

\author{
Kouao J. Koffi ${ }^{1 *}$, Dominique Champluvier ${ }^{2}$, Danho F. R. Neuba ${ }^{3}$, Charles De Canniere ${ }^{1}$, Traoré Dossahoua ${ }^{4}$, \\ Jean LEJOLY ${ }^{1}$, Elmar ROBBRECHT ${ }^{2}$ \& Jan BOGAERT ${ }^{1}$
}
${ }^{1}$ Université libre de Bruxelles, Laboratoire d'Ecologie du Paysage, Avenue F. D. Roosevelt 50 - CP 169, B-1050 Bruxelles, Belgique. 2 Jardin Botanique National de Belgique, Domaine de Bouchout, B-1860 Meise.
${ }^{3}$ Université libre de Bruxelles, Service de Botanique Systématique et de Phytosociologie, Avenue F. D. Roosevelt 50 - CP 169, B-1050 Bruxelles, Belgique.
${ }^{4}$ Université d'Abidjan-Cocody, Faculté des Sciences et Techniques, Département de Botanique et Biologie Végétale,
22 B.P. 582, Abidjan 22, Côte d'lvoire.
*Auteur pour les correspondances: (E-mail: kkoffi@ulb.ac.be)
Recu le 21-06-2006, accepté le 15-02-2008.

\begin{abstract}
Résumé
Cette étude a pour but d'analyser les modèles de distribution spatiale des Acanthaceae, récoltées en République Démocratique du Congo, au Rwanda et au Burundi et de comparer leur répartition spatiale avec les systèmes phytogéographiques qui couvrent cette zone. Elle porte sur 9181 échantillons regroupés en 48 genres et 310 espèces. Un système d'information géographique a été utilisé pour réaliser des cartes de distribution de chaque espèce. La méthode multi variée de classification, appuyée par deux indices de structure spatiale (équitabilité et fragmentation) a permis de stratifier la zone d'étude afin de permettre une comparaison visuelle avec les systèmes phytogéographiques définis par Robyns, White et Ndjele. On note une large distribution spatiale pour certaines espèces sur l'ensemble du territoire, alors que d'autres sont inféodées à certains territoires phytogéographiques bien déterminés. Les savanes situées au Sud de l'équateur contiennent des sites plus riches en Acanthaceae que celles situées au Nord. Les centres d'endémisme Guinéo-Congolais, Zambézien et Afromontagnard pourraient être les sources de migration des taxons. Les Acanthaceae suivent relativement bien le système phytogéographique de White et sont utilisables en tant que bioindicatrices afin de rapprocher la distribution spatiale des communautés végétales décrites par ce système.
\end{abstract}

Mots clés: phytogéographie, analyse spatiale, Acanthaceae, Afrique Centrale, district, secteur

\section{Abstract \\ Analysis of the spatial distribution of Acanthaceae in Central Africa and comparison with the phytogeographic theories of Robyns, White and Ndjele.}

By means of 9181 samples representing 48 genus and 310 species, the spatial distribution models of the Acanthaceae family in Central Africa (Democratic Republic of Congo, Rwanda and Burundi) were analysed. Consequently, a comparison to existing phytogeographic systems for the study region was executed. Spatial distribution maps for each species were created using a geographic information system. Subdivision of the study area was realised using a multivariate classification analysis. Using two indices of spatial pattern (fragmentation, class size evenness), the classification result was compared to the phytogeographic zones defined by Robyns, White and Ndjele. While certain species showed a distribution throughout the study area, the presence of other species was found to be bound to one single phytogeographic territory in particular, or to a restricted number of them. The savannas situated in the southern part of the study area contained more Acanthaceae species than the zones situated north of the equator. The Guineo-Congolian, the Zambezian and the Afromontane centre of endemism are suggested to be the origins of taxon migration. Since it was observed that the spatial pattern of the Acanthaceae species corresponded up a certain extent to the phytogeographic system proposed by White, it can be concluded that the Acanthaceae could be used as bio-indicators to proxy the spatial distribution of plant communities as described by this system.

Key Words: phytogeography, spatial analysis, Acanthaceae, Central Africa, district, sector. 


\section{Introduction}

La phytogéographie est une science qui étudie la répartition des espèces à la surface du globe (Schnell, 1971). Elle repose sur des bases cartographiques dont l'outil principal est la carte de répartition. Celle-ci permet de connaître avec précision la distribution géographique d'une espèce ainsi que l'importance des divers facteurs qui la contrôlent (Lebrun, 2001). En effet, les cartes de répartition spatiale indiquent les zones de fortes diversités spécifiques appelées hotspots, la diversité spatiale des espèces et les zones d'endémisme. Elles constituent donc un outil principal pour la gestion et la conservation des espèces. Elles permettent également de poser des hypothèses solides sur l'âge, l'origine géographique, la vitesse d'évolution et les voies de migration des taxons. II est même possible, à partir de la distribution des espèces de mettre en évidence les différentes aires végétales et le découpage de la planète en unités phytogéographiques (régions, districts, secteurs, etc.). Plusieurs études portant sur les subdivisions phytogéographiques fondées sur des données et arguments physionomiques et endémiques ont été réalisées en Afrique Centrale. Robyns (1948) a proposé un système basé sur les aspects de la végétation et le bioclimat (hauteur des précipitations, durée de la saison sèche), en subdivisant la République Démocratique du Congo (R.D. Congo), le Rwanda et le Burundi en 11 districts phytogéographiques (Fig. 1a). White (1979, 1986), en se basant en grande partie sur la distribution des Ebenaceae, a subdivisé l'Afrique et Madagascar en 20 entités régionales dont six recouvrent la R.D. Congo, le Rwanda et le Burundi (Fig. 1b). Ndjele (1988), après avoir également perfectionné le système de Robyns (1948), a également proposé un nouveau système de classification phytogéographique de la R.D. Congo en six régions, sept domaines, 13 secteurs (Fig. 1c) et 24 districts phytogéographiques. Le but de notre étude est, d'une part, d'analyser les modèles de distribution spatiale des Acanthaceae de la R.D. Congo, du Rwanda et du Burundi et d'autre part, de rechercher les convergences et divergences de ces modèles avec les différents systèmes phytogéographiques proposés ci-dessus. Le choix des Acanthaceae se justifie par le fait que c'est une famille qui a été largement étudiée de
1888 à 2001 par environ 427 collecteurs au cours des missions organisées en R.D. Congo, au Rwanda et au Burundi. La plupart des échantillons de ces différentes récoltes sont conservés dans l'herbier du Jardin Botanique National de Meise (BR) en Belgique. Cette famille a, en outre, fait récemment l'objet d'une révision systématique afin de contribuer à la réalisation de la flore des Acanthaceae de la R.D. Congo, du Rwanda et du Burundi. Pour mener cette étude, une base de données des Acanthaceae a été créée et chaque espèce a été cartographiée (Koffi, 2005). Les modèles de distribution spatiale ont été comparés par des valeurs quantitatives et physionomiques avec les systèmes phytogéographiques de Robyns (1948), White (1979, 1986) et de Ndjele (1988). Nous recherchons par cette méthodologie à mettre en évidence le système qui reflèterait le mieux la distribution générale des Acanthaceae.

\section{Matériel et méthodes}

\subsection{Matériels d'étude}

Le matériel ayant servi à la réalisation de cette étude est constitué de 9181 spécimens d'herbier du Jardin Botanique National de Belgique (BR). II s'agit d'un matériel révisé au cours des travaux sur la flore de l'Afrique Centrale, plus précisément la R.D. Congo, du Burundi et du Rwanda. Cet ensemble géographique est compris dans le domaine équatorial, subéquatorial et dans la zone tropicale dans ses bordures Nord et Sud au-delà du 8ème degré (Van Chi Bonnardel, 1973).

\subsection{Méthodes d'étude}

2.2.1. Traitement de la base de données et choix de la taille des mailles

La base de données de cette étude a été réalisée dans BRAHMS 5 (Anonyme, 2004). Les espèces rudérales, aquatiques et cultivées n'ont pas été prises en compte dans l'étude parce que les résultats des analyses peuvent être influencés par leur distribution spatiale déterminée par des facteurs non écologiques. Le système de maillage de Denys (1980), Lebrun (1981), Ndjele (1988) et de Crisp et al. (2001) a été adopté. Sur la base d'une comparaison de mailles de différentes tailles $\left(0,20^{\circ}-0,25^{\circ}-0,50^{\circ}-0,75^{\circ}\right.$ - 
$1,00^{\circ}-1,25^{\circ}-1,50^{\circ}-1,75^{\circ}-2,00^{\circ}$ ), la taille qui permettait d'avoir moins de $10 \%$ de mailles vides a été retenu. En effet la courbe de la Fig. 2a qui représente le pourcentage des mailles vides en fonction de la taille de celle-ci, décroît pour se stabiliser à environ $10 \%$. Cette valeur correspond à des quadrats de $1,50^{\circ}$. Dans la suite du travail, des quadrats de $1,50^{\circ} \times 1,50^{\circ}$ numérotés de 1 à 118 (Fig. 2b) sont retenus pour l'analyse de classification. Arcview GIS 3.3 (www.esri.com) a servi à réaliser toutes les cartes nécessaires à la réalisation de ce travail.
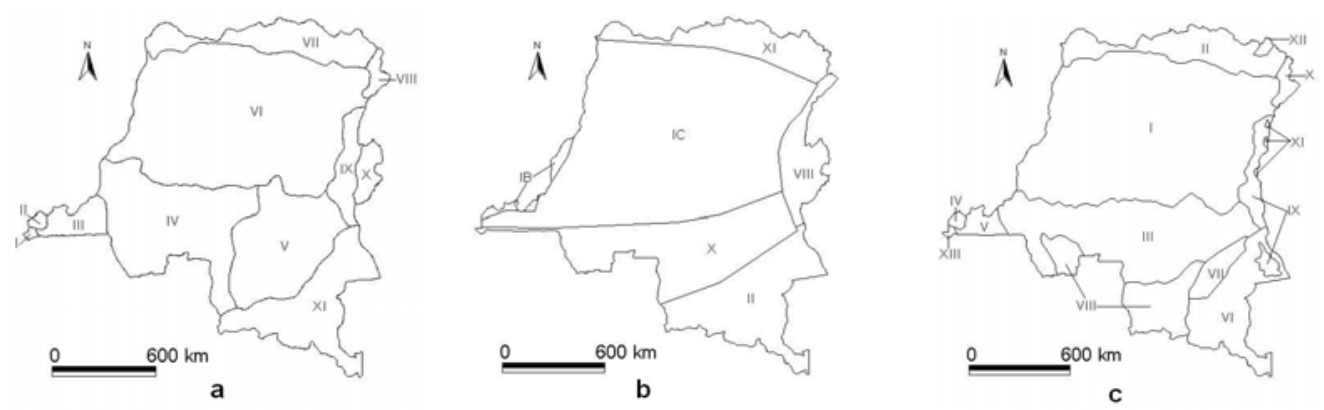

Figure 1: Différents systèmes phytogéographiques de la République Démocratique du Congo (R.D. Congo), du Rwanda et du Burundi:

a) Districts phytogéographiques de Robyns (1948). I: Côtier; II: Mayumbe; III: Bas-Congo; IV: Kasai; V: Bas-Katanga; VI: Forestier central; VII: Ubangi-Uele; VIII: Lac-Albert; IX: Lacs Edouard et Kivu; X: Ruanda-Urundi; XI: Haut-Katanga.

b) Entités phytogéographiques de White $(1979,1986)$ couvrant la R.D. Congo, le Rwanda et le Burundi. l: centre régional d'endémisme Guinéo-Congolais (IB : sous-centre Guinéen inférieur; IC : sous-centre Congolais); II: centre régional d'endémisme Zambézien; VIII: centre régional d'endémisme Afromontagnard; X: zone de transition régionale Guinéo-Congolaise/Zambézienne; XI: zone de transition régionale Guinéo-Congolaise/Soudanienne.

c) Secteurs phytogéographiques de Ndjele (1988). I: secteur Forestier central; II: secteur de transition Congolo-Soudanien; III: secteur de transition Congolo-Zambézien; IV: secteur Forestier du Mayumbe; V: secteur de transition Bas-Guinéo-Zambézien; VI: secteur Bemba; VII: secteur du Lualaba; VIII: secteur Lunda; IX: secteur des Montagnes; X: secteur du Lac Mobutu; XI: secteur des Hautes plaines du Kivu; XII: secteur Soudanien Méridional; XIII: secteur Sud-Atlantique du littoral Guinéen.

Les traits indiquent les limites des territoires phytogéographiques.
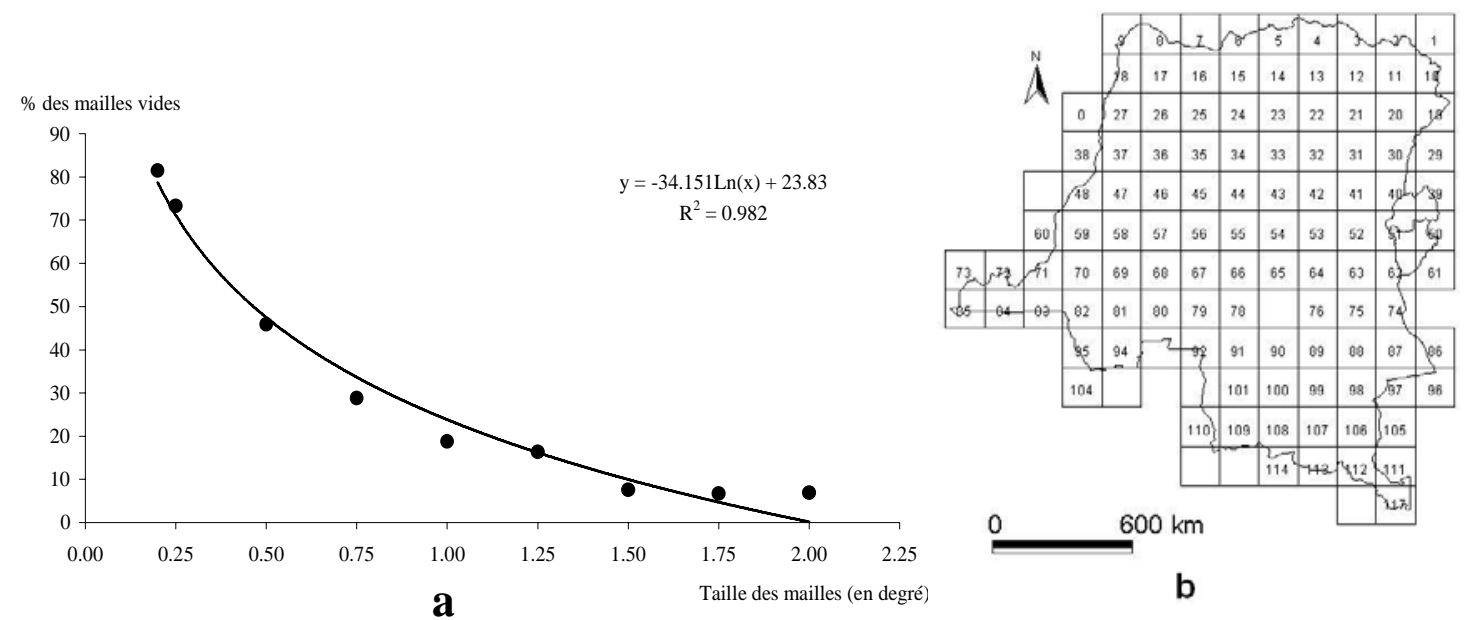

Figure 2: Choix de la taille des mailles:

a) taille des mailles en fonction du pourcentage (\%) des mailles vides;

b) Site d'étude superposé de mailles de $1,5^{\circ}$.

La courbe en trait continu est la courbe de tendance. 


\subsubsection{Analyse de classification et cartographie de la structure spatiale des Acanthaceae}

Une matrice présence/absence a été constituée pour permettre de comparer la composition floristique des mailles à l'aide des indices de similarité et de différentes stratégies de regroupement (nearest neighbor, farthest neighbor, unweighted centroid, unweighted pair group average, weighted pair group average, weighted centroid). Tous les indices de similarité disponibles dans le logiciel MVSP (Multi-Variate Statistical Package) version 3.1 (Kovach, 2003) et qui tiennent compte de la matrice présence/ absence ont été utilisés. II s'agit de : Sørensen's coefficient, Jaccard's coefficient, Simple matching coefficient, Yule coefficient, Nei and Li's coefficient, Baroni-Urbani Buser coefficient. Tous ces indices ont été utilisés dans le but de trouver celui qui permet d'obtenir une carte semblable aux cartes préexistantes. Pour chaque indice de similarité, la matrice est soumise aux algorithmes de classification hiérarchique ci-dessus cités. Pour la confection des cartes, les dendrogrammes permettant d'obtenir un nombre de groupes identiques aux subdivisions de Robyns (1948), de White $(1979,1986)$ et de Ndjele (1988) sont retenus. Toutefois, il faut signaler que pour des raisons pratiques, certaines règles ont été suivies. En effet, pour les carrés se trouvant dans plus de deux subdivisions, le comptage est attribué à la subdivision qui a la plus grande surface et les subdivisions très petites ou confinées dans une autre subdivision sont réunies pour en faire une. Par conséquent, avec la carte de Robyns (1948), les Districts I \& II fusionnent pour donner 10 Districts au lieu de 11 ; avec la carte de Ndjele (1988), les Secteurs IV \& XIII fusionnent ainsi que les Secteurs IX \& XI pour donner 11 Secteurs au lieu de 13. Les cartes représentant ces différents groupes, réalisées à l'aide d'Arcview, sont ensuite comparées de façon quantitative et visuelle à celles des auteurs cités en considérant deux indices de structure spatiale: l'équitabilité et la fragmentation. L'équitabilité ou la régularité est donnée par la formule de Pielou (1975):

$$
R=-\frac{\sum_{i=1}^{n} p_{i} \log p_{i}}{\log n}
$$

avec $p_{i}$-la proportion des mailles de chaque zone et $n$-le nombre de subdivisions. L'indice de fragmentation $F$ mesure l'agrégation des mailles. II permet de déterminer le degré de fragmentation d'une carte (Johnson, 1995; Bogaert et al., 2002). II est calculé comme suit:

$$
F=\frac{k-1}{m-1} ;
$$

avec $k$-le nombre d'agrégats de mailles formé $(k=1)$ et $m$-le nombre de mailles, $0=F=1$. Lorsque $F=0$, la carte a une structure agrégée $(k=1)$ et quand $F=1$, la structure est fragmentée $(k=m)$.

\section{Résultats}

\subsection{Analyse floristique et densité de prospection}

Neuf mille cent quatre vingt un échantillons d'Acanthaceae ont été encodés et regroupés en 48 genres, 310 espèces et 6362 localités. Pour 19 espèces, plus de 100 échantillons ont été prélevés, pour 35 espèces entre 99 et 50 échantillons, pour 141 espèces entre 49 et 10 échantillons, pour 89 espèces entre neuf et trois échantillons et pour 25 espèces entre un et deux échantillons. Les espèces les plus échantillonnées sont : Asystasia gangetica (Lin.) T. Anderson subsp. micrantha (Nees) Ensermu (401 échantillons), Whitfieldia elongata (P. Beauv.) De Wild. et Th. Dur. (265 échantillons), Hypoestes forskalei (Vahl.) Soland. ex Roem. et Schult. (218 échantillons) et Pseuderanthemum ludovicianum (Büttn.) Lindau (212 échantillons). D'autres par contre ne sont représentées que par un à trois échantillons. C'est le cas de: Barleria elegans S. Moore, Lepidagathis peniculifera S. Moore, Ruellia togoensis (Lindau) Heine. Les genres qui regroupent les plus grands nombres d'échantillons sont: Justicia Lin. avec 1595 échantillons $(17,1 \%$ des échantillons; 58 espèces), Thunbergia Retz avec 813 échantillons $(8,7 \%$ des échantillons; 36 espèces), et Asystasia Blume avec 638 échantillons $(6,8 \%$ des échantillons; 11 espèces). La carte de distribution des 9181 échantillons d'Acanthaceae (Fig. 3) montre que ces végétaux colonisent aussi bien les biotopes de très basses altitudes (plaine côtière de l'Océan 
Atlantique) que ceux des hautes altitudes (au dessus de $2000 \mathrm{~m}$ de la Région Afromontagnarde). Elle met également en évidence les niveaux de prospection. L'exploration de la zone d'étude n'est pas uniforme. Certaines zones ont été plus densément prospectée (Boma, Kinshasa, Lubumbashi, Bukavu, Goma, Bunia, Kisangani Rwanda-Burundi), alors que d'autres l'ont été partiellement ou pas du tout.

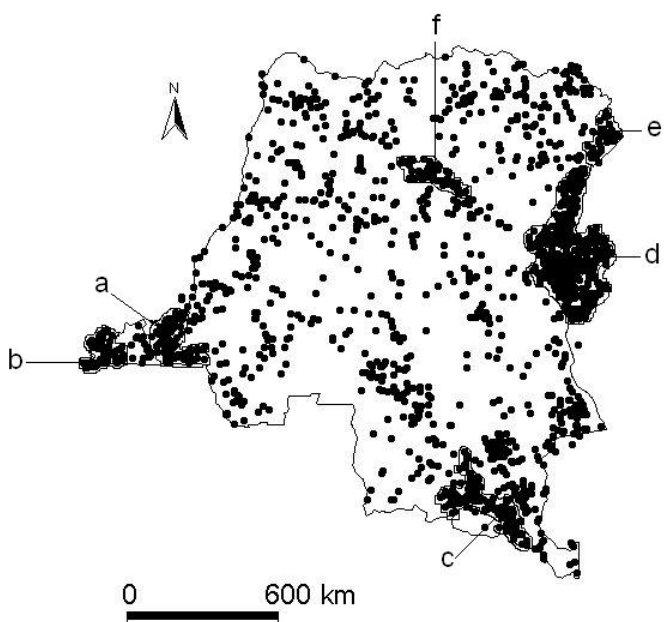

Figure 3: Intensité de l'exploration floristique de la République Démocratique du Congo (R.D. Congo), du Rwanda et du Burundi:

a: Kinshasa;

b: Boma;

c: Lubumbashi, Musoshi;

d: Bukavu, Goma, Rwanda, Burundi;

e: Bunia;

f: Kisangani.

Le symbole (-) indique les lieux de récolte des échantillons et les zones encadrées sont les plus prospectées.

\subsection{Analyse des modèles de distribution}

Les cartes de distribution de chaque espèce mettent en évidence les différents modèles de distribution des Acanthaceae en R.D. Congo, au Rwanda et au Burundi. En effet, on note pour certaines espèces une large distribution spatiale sur l'ensemble du territoire. C'est le cas de Whitfieldia elongata (Fig. 4a), récoltée dans les neuf districts phytogéographiques de Robyns (1948). Par contre, d'autres sont inféodées à certains territoires bien déterminés. C'est le cas de Hygrophila pilosa Burkill (Fig. 4b), récoltée uniquement dans le district du Haut-Katanga de Robyns (1948). Les Fig. $4 c$ et $4 d$ montrent que l'aire de distribution de cette espèce se limite aussi au centre régional d'endémisme Zambézien de White $(1979,1986)$ et au secteur Bemba de Ndjele (1988). D'autres espèces bien qu'étant abondamment récoltées dans un territoire, ont quelques spécimens dans les territoires phytogéographiques voisins. C'est le cas de Asystasia vogeliana Benth. (Fig. 4e), abondamment récoltée dans le district du Forestier central, mais quelques spécimens ont été rencontrés dans le district Ubangi-Uele, le district des Lacs Edouard et Kivu, le district du Bas-Katanga et dans le district du Kasai de Robyns (1948).

\subsection{Présence des Acanthaceae dans les systèmes phytogéographiques}

Au regard des trois spectres phytogéographiques (Fig. 5), le maximum d'espèces d'Acanthaceae a été récolté dans le district XI (Haut-Katanga) de Robyns (1948) qui correspond au centre régional d'endémisme Zambézien (II) de White (1979, 1986) et au secteur Bemba (VI) décrit par Ndjele (1988). Le district IX (Lacs Edouard et Kivu) de Robyns (1948), correspondant au centre régional d'endémisme morcelé Afromontagnard (VIII) de White $(1979,1986)$, vient en deuxième position. II est suivi du district VI (Forestier central) de Robyns (1948), également appelé centre régional d'endémisme Guinéo-Congolais (I) par White $(1979,1986)$. La superposition des cartes de tous les échantillons montre que les espèces ont tendance à migrer vers les autres districts ou secteurs phytogéographiques à partir de ces trois foyers qui correspondent à la zone de savane, la zone de montagne et la zone de forêt. Ils pourraient être considérés comme les sources de migration de certaines espèces. Le district du Haut-Katanga ou centre régional d'endémisme Zambézien est le plus diversifié en Acanthaceae (Fig. 5). Le système phytogéographique qui regorge le plus d'espèces inféodées aux territoires déterminés est celui de White $(1979,1986)$ avec $37,74 \%$, suivi de Ndjele (1988) et Robyns (1948) avec respectivement $27,74 \%$ et $26,16 \%$ du nombre total d'espèce. Dans toutes les entités phytogéographiques de White $(1979,1986)$, tous les centres régionaux d'endémisme sont caractérisés par les Acanthaceae. 


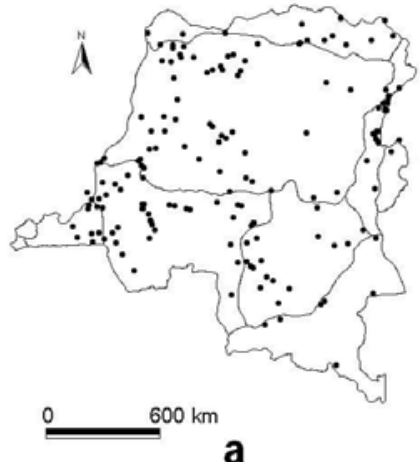

a
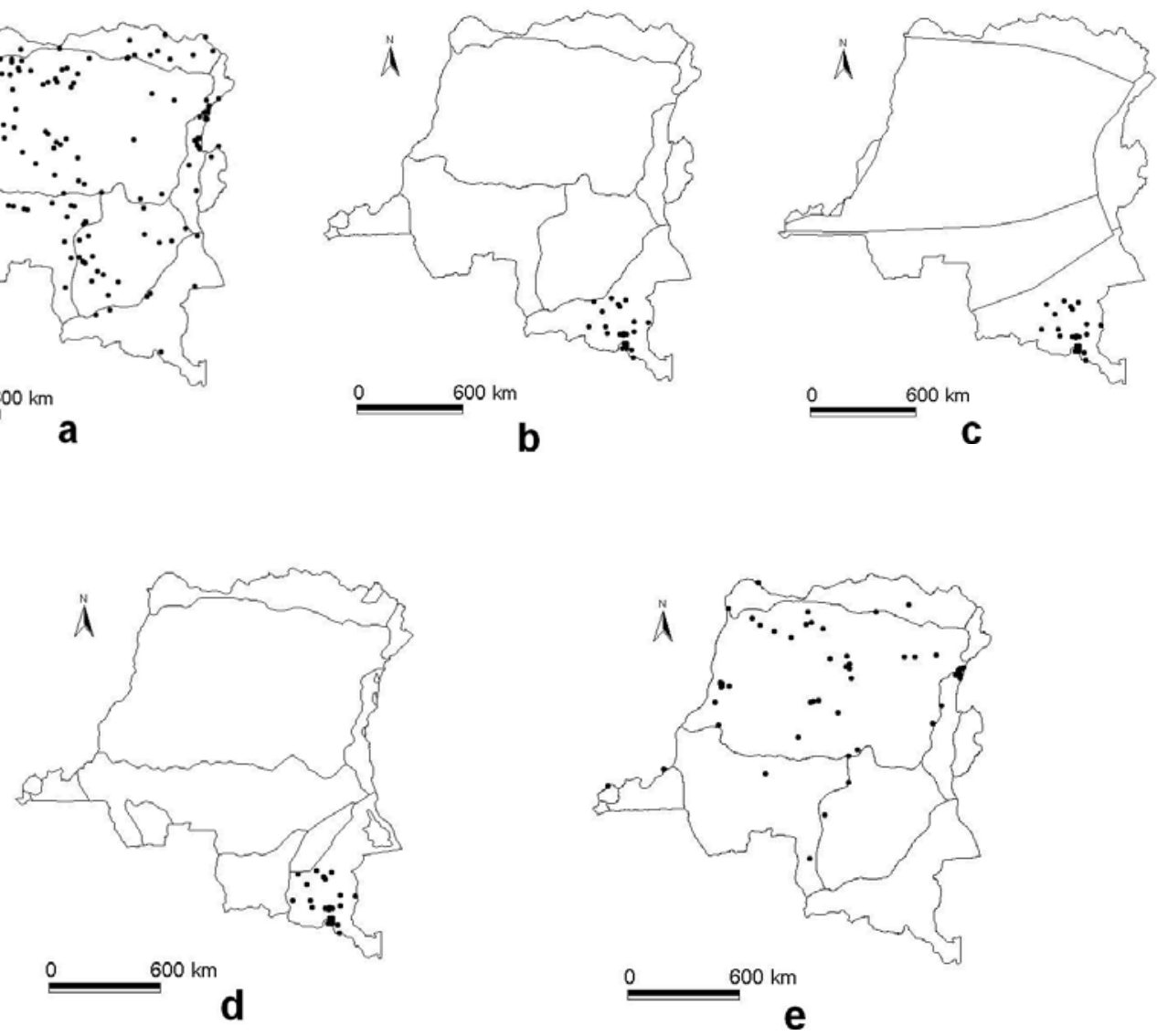

Figure 4: Exemples de distributions par rapport aux systèmes phytogéographiques de la République Démocratique du Congo (R.D. Congo), du Rwanda et du Burundi:

a) Whitfieldia elongata (P. Beauv.) De Wild. et Th. Dur, récoltée dans les neuf districts phytogéographiques de Robyns (1948);

b) Hygrophila pilosa Burkill, rencontrée uniquement dans le district du Haut-Katanga de Robyns (1948);

c) Hygrophila pilosa Burkill, récoltée dans le centre régional d'endémisme Zambézien de White (1979, 1986);

d) Hygrophila pilosa Burkill, récoltée dans le secteur Bemba de Ndjele (1988);

e) Asystasia vogeliana Benth abondamment récoltée dans le district du Forestier central avec quelques échantillons dans les districts voisins de Robyns (1948).

Le symbole (') indique les lieux de récolte des échantillons et les traits les limites des districts phytogéographiques. 

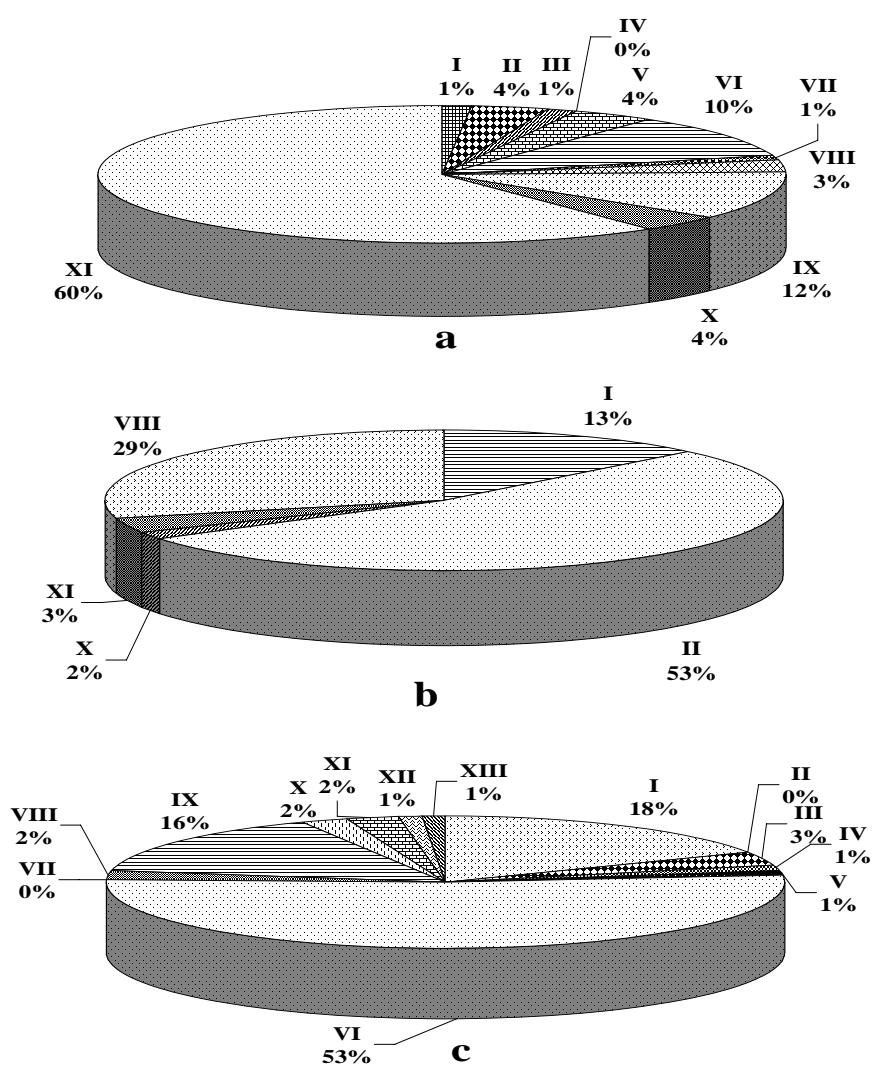

Figure 5: Spectre phytogéographique des Acanthaceae et richesse de la flore par rapport aux différents systèmes phytogéographiques de la République Démocratique du Congo (R.D. Congo), du Rwanda et du Burundi:

a) Robyns (1948);

b) White $(1979,1986)$

c) Ndjele (1988).

Le pourcentage se rapporte à la flore des Acanthaceae récoltées en R.D. Congo, au Rwanda et au Burundi. Voir Fig. 1 pour les codes des territoires phytogéographiques.

\subsection{Cartographie de la structure spatiale des Acanthaceae}

La comparaison visuelle a été faite entre les cartes de Robyns (1948), de White $(1979,1986)$ et de Ndjele (1988) et celles obtenues avec les indices de similarité qui ont les indices d'équitabilité et de fragmentation proches. En effet, l'indice d'équitabilité de la carte de Robyns (1948) est 0,83 et son indice de fragmentation est 0,09 . La carte qui a son indice d'équitabilité $(0,74)$ et son indice de fragmentation $(0,20)$ proches de celle de Robyns (1948) est la carte obtenue avec l'indice de similarité de Baroni et la stratégie de groupement unweighted centroid
(Fig. 6a). L'indice d'équitabilité de la carte de White $(1979,1986)$ est 0,87 et son indice de fragmentation est 0,04 . La carte qui a son indice d'équitabilité $(0,64)$ et son indice de fragmentation $(0,08)$ proches de celle de White $(1979,1986)$ est la carte obtenue avec l'indice de similarité de Baroni et la stratégie de groupement weighted centroid (Fig. 6b). Enfin, l'indice d'équitabilité de la carte de Ndjele (1988) est 0,79 et son indice de fragmentation est 0,11 ; la carte qui a son indice d'équitabilité $(0,77)$ et son indice de fragmentation $(0,20)$ proches de celle de la carte de Ndjele (1988) est la carte obtenue avec l'indice de similarité de Baroni et la stratégie de groupement unweighted centroid (Fig. 6c). 


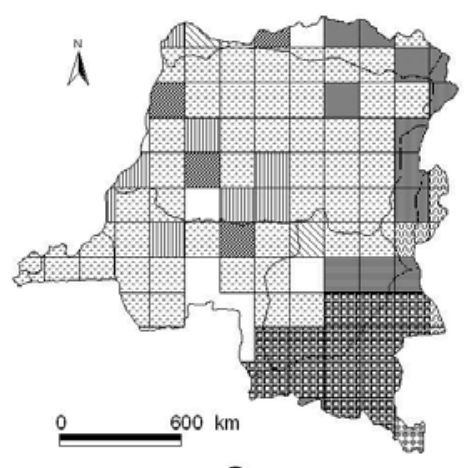

a

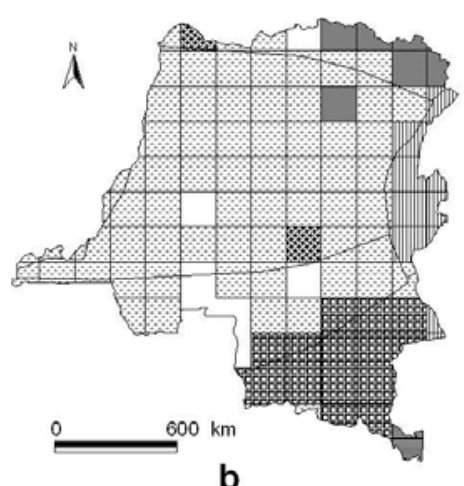

b

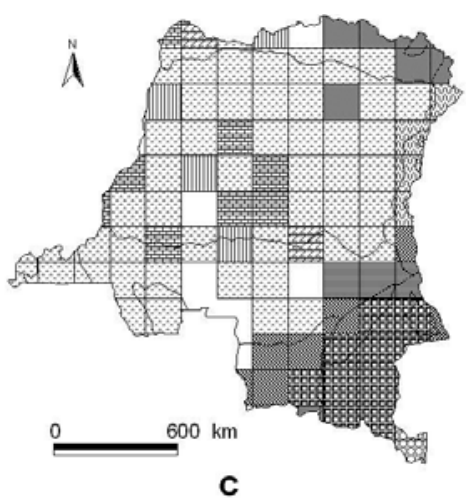

c

Figure 6: Cartes utilisées dans cette étude pour la comparaison visuelle des systèmes phytogéographiques de la République Démocratique du Congo (R.D. Congo), du Rwanda et du Burundi :

a) carte obtenue avec l'indice de similarité de Baroni et la stratégie de groupement unweighted centroid pour la comparaison avec les districts phytogéographiques de Robyns (1948);

b) carte obtenue avec l'indice de similarité de Baroni et la stratégie de groupement weighted centroid pour la comparaison avec les entités phytogéographiques de White (1979, 1986);

c) carte obtenue avec l'indice de similarité de Baroni et la stratégie de groupement unweighted centroid pour la comparaison avec les secteurs phytogéographiques de Ndjele (1988).

\section{Discussion}

Les régions les mieux connues floristiquement sont celles situées le long de la côte (Boma), les zones environnantes des centres d'activités et des institutions de recherche et des universités (Kinshasa, Lubumbashi, Bukavu, Kisangani) et les zones à climat tempéré (Rwanda-Burundi). En dehors de ces zones, de grands espaces très diversifiés en végétation naturelle ne sont pas explorés ou ne le sont que très partiellement. Ces constats sont conformes à ceux de Hepper (1979) qui stipule qu'en R.D. Congo, au Rwanda et au Burundi, il y a des régions bien connues, des régions moyennement connues et des régions mal connues. Les espèces qui ont été récoltées presque sur l'ensemble de la zone d'étude sont des espèces qui ont une large amplitude écologique. Elles sont capables de s'adapter aux facteurs édaphiques et abiotiques divergents. C'est le cas de Whitfieldia elongata. Par contre, celles récoltées dans des endroits bien délimités sont des espèces qui ont vraisemblablement une amplitude écologique plus restreinte. C'est le cas Thunbergia manikensis De Wild. D'après Schnell (1971), beaucoup d'espèces ont eu une aire beaucoup plus vaste, mais n'ont pu suivre les changements climatiques relativement rapides, ce qui pourrait expliquer la présence de certaines espèces dans des zones restreintes. D'autres encore bien qu'étant abondamment récoltées dans un territoire, ont quelques spécimens dans les territoires phytogéographiques voisins. C'est le cas de Acanthus montanus (Nees) T. Anderson. Ces dernières peuvent êtres qualifiées d'espèces de transition. Dans le cadre de la conservation, la valeur indicatrice des Acanthaceae n'est pas constante dans tous les secteurs d'étude en raison de la variation du nombre de représentation par espèce. L'utilisation d'espèces avec un nombre restreint d'échantillons comme Barleria elegans, Ruellia togoensis et Lepidagathis peniculifera pour caractériser les territoires phytogéographiques devrait donc être aperçue avec prudence.

Le district XI (Haut-Katanga) de Robyns (1948) ou centre régional d'endémisme zambézien par White $(1979,1986)$ et secteur VI (secteur Bemba) par Ndjele (1988) est la région qui regorge le maximum d'espèces. Ces résultats sont conformes aux subdivisions de Lebrun (1960, 1976) et Ozenda (1982). En effet, il apparaît dans ces travaux que le grand centre de spéciation de la flore africaine est situé dans la région Zambézienne et plus précisément dans le 
domaine de l'élément floral BangweoloKatangais. Les mêmes résultats ont été obtenus avec la flore des Eriocaulaceae d'Afrique centrale (Kimpouni, 1993). Au niveau générique, le centre régional d'endémisme zambézien est aussi le centre de spéciation des genres Thunbergia et Justicia. En effet, Ndjele (1988) a signalé dans ses travaux que Thunbergia faisait partie des principaux genres rencontrés dans cette région.

En général, aucune carte n'a mis entièrement en évidence les différents territoires phytogéographiques proposés par Robyns (1948), White $(1979,1986)$ et Ndjele (1988). Ceci pourrait s'expliquer par le fait que la récolte des échantillons n'est pas uniforme. Certaines zones ont été mieux prospectées que d'autres (Fig. 3). Néanmoins, la carte obtenue avec l'indice de similarité de Baroni et la stratégie de groupement unweighted centroid (Fig. 6a), révèle les districts des Lacs Edouard et Kivu (IX), du Ruanda-Urundi (X) et du Haut-Katanga (XI) déterminés par Robyns (1948). Quatre zones déterminées par White $(1979,1986)$ ont été mises en évidence par la carte obtenue avec l'indice de similarité de Baroni et la stratégie de groupement weighted centroid. II s'agit du centre régional d'endémisme Zambézien (II), du centre régional d'endémisme Afromontagnard (VIII), de la zone de transition régionale GuinéoCongolaise/Soudanienne $(\mathrm{XI})$ et du centre régional d'endémisme Guinéo-Congolais (I) (Fig. 6b). La carte obtenue avec l'indice de similarité de Baroni et la stratégie de groupement unweighted centroid, met en relief le secteur Bemba (VI), le secteur Lunda (VIII), le secteur des Montagnes (IX) et le secteur Soudanien Méridional (XII) décrits par Ndjele (1988) (Fig. 6c). Les Acanthaceae suivent mieux les subdivisions de White $(1979,1986)$. Ces subdivisions couvrant la R.D. Congo, le Rwanda et le Burundi sont, en effet, caractérisées par les Acanthaceae qui ont leur distribution spatiale limitée à ces entités. Le district IV (Kasai) de Robyns (1948), les secteurs II (secteur de transition Congolo-Soudanien) et VII (secteur du Lualaba) de Ndjele (1988) ne sont pas marqués par les Acanthaceae. Ce qui pourrait s'expliquer par le fait que cette zone a été insuffisamment explorée à cause de son inaccessibilité. L'indice de similarité Baroni-Urbani Buser avec sa stratégie de groupement Median ou Centroid, a été l'indice qui a le mieux mis en évidence les différents territoires phytogéographiques comparés.

\section{Conclusion}

La carte de distribution de tous les échantillons a revelé les niveaux de prospection botanique en R.D. Congo, au Rwanda et au Burundi. Les zones les mieux prospectées sont celles situées aux alentours des centres d'activités et des institutions de recherche. Les savanes situées au Sud et les forêts claires contiguës, possèdent des sites plus riches en Acanthaceae que celles situées au Nord de l'équateur. Les Acanthaceae convergent plus avec le système phytogéographique de White (1979, 1986) que ceux proposés par Robyns (1948) et Ndjele (1988). Certaines espèces ont une large amplitude écologique, d'autres par contre sont inféodées à un type particulier d'habitats. Les espèces limitées aux régions géographiques restreintes sont des espèces menacées d'extinction. Elles pourraient être utilisées dans une première approche comme espèces indicatrices de certains systèmes phytogéographiques. Par conséquent, elles doivent être conservées ainsi que leurs habitats respectifs. Pour des entités phytogéographiques particulières, aucune espèce caractéristique n'a été rencontrée, ce qui indique que l'approche courante doit être raffinée. La comparaison avec d'autres familles et la confrontation avec d'autres théories et concepts phytogéographiques devraient être effectuées avant que des conclusions plus profondes ne soient tirées.

\section{Remerciements}

Les auteurs remercient le gouvernement de Côte d'Ivoire pour la bourse de formation de Kouao Jean Koffi. Cette recherche a été financée par le FNRS (crédit 1.5.028.05) et I'ULB (Crédit extraordinaire de recherche).

\section{Références citées}

Anonyme, 2004. Botanical Research And Herbarium Management System. University of Oxford. 5.58-1-EJM053-10 Feb. 2005.

Bogaert J., Zhou L., Tucker C. J., Myneni R. B. et Ceulemans R., 2002. Evidence for a persistent and extensive greening trend in Eurasia inferred from satellite vegetation index data. Journ. Geophysic. Res. D11, 10.1029/2001JD001075 (107). 14 pp. 
Crisp M. D., Laffan S., Linder H. P. et Monro A. 2001. Endemism in the Australian flora. Journ. Biogeogr. (28):183-198.

Denys E., 1980. A tentative pytogeographical division of tropical Africa based on a mathematical analysis of distribution maps. Bull. Jard. Bot. Nat. Belg. 50 (3/4): 465-504.

Hepper, N.F. 1979. Deuxième édition de la carte du degré d'exploration floristique de l'Afrique au sud du Sahara. in G. Kunkel Eds., Proceedings of the IXth 22 plenary meeting of the Association pour l'Etude Taxonomique de la Flore d'Afrique Tropicale (AETFAT), 18-23 March 1978, Las Palmas de Gran Canaria. pp 157-162.

Johnsson K., 1995. Fragmentation index as a region based GIS operator. Int. J. Geogr. Inf. Syst. (9): 211-220.

Kimpouni V., 1993. Les Eriocaulaceae d'Afrique centrale (Congo, Zaïre, Rwanda, Burundi). Thèse. Faculté des Sciences, Université libre de Bruxelles, Belgique. 588 pp.

Koffi K. J., 2005. Analyse des structures spatiales des données de distribution phytogéographique en Afrique centrale. Mémoire de DEA. Faculté des Sciences, Université libre de Bruxelles, Belgique. 190 pp.

Kovach W.L., 2003. Multi-Variate Statistical Package for Windows. User'Manuel. Version 3.1. Publ. Kov. Comp. Serv. Pentraeth, Wales, U.K. $137 \mathrm{pp}$.

Lebrun J., 1960. Sur une méthode de délimitation des horizons et étages de végétation des montagnes du Congo Oriental. Bull. Jard. Bot. (30): 75-94.
Lebrun J. P., 1976. Richesse spécifique de la flore vasculaire des divers pays ou régions d'Afrique. Candollea (31):11-15.

Lebrun J. P., 2001. Introduction à la flore d'Afrique. Cirad. Ibis Press. 155 pp.

Ndjele M.B., 1988. Les éléments phytogéographiques endémiques dans la flore vasculaire du Zaïre. Thèse. Faculté des Sciences, Université libre de Bruxelles, Belgique. 528 pp.

Ozenda P., 1982. Les végétaux dans la biosphère. Eds. Dion, Paris. 431 pp.

Pielou E. C., 1975. Ecological Diversity. John Wiley et Sons, New York. 165 pp.

Robyns W., 1948. Les territoires phytogéographiques du Congo Belge et du Ruanda-Urundi.Atlas général du Congo Belge. Inst. Roy. Col. Belge, Bruxelles, 10 pp.

Schnell R., 1971. Introduction à la phytogéographie des pays tropicaux: les milieux - les groupements végétaux. Géobiologie Ecologie Aménagement. (2): 951 pp.

Sys C., 1960. Carte des sols et de la végétation du Congo Belge et du Rwanda-Burundi. Notice explicative de la carte des sols du Congo Belge et du Rwanda-Burundi. Publ. I.N.E.A.C., Bruxelles. 84 pp.

Van Chi Bonnardel R., 1973. Grand Atlas du continent Africain. Ed. Jeune Afrique, Paris, 336 pp.

White F., 1979. The guineo-congolian region and its relationships to other phytochoria. Bull. Jard. Bot. Nat. Belg. (49): 11-55.

White F., 1986. La végétation de l'Afrique. Mémoire accompagnant la carte de végétation de l'Afrique, Unesco/Aetfat/Unso. 384 pp. 